\title{
Corrigendum to "Breast Cancer Survival and Incidence: 10 Years Cancer Registry Data in the Northwest, Iran"
}

\author{
Roya Dolatkhah $\mathbb{D}^{1},{ }^{1}$ Mohammad Hossein Somi, ${ }^{2}$ Mohammad Asghari Jafarabadi, ${ }^{3}$ \\ Mehrnaz Hosseinalifam, ${ }^{4}$ Sepideh Sepahi, ${ }^{5}$ Mina Belalzadeh, ${ }^{5}$ Marzieh Nezamdoust, ${ }^{5}$ \\ and Saeed Dastgiri $\mathbb{C}^{6}$ \\ ${ }^{1}$ Hematology and Oncology Research Center, Tabriz University of Medical Sciences, Tabriz, Iran \\ ${ }^{2}$ Liver and Gastrointestinal Diseases Research Center, Tabriz University of Medical Sciences, Tabriz, Iran \\ ${ }^{3}$ Road Traffic Injury Research Center, Tabriz University of Medical Sciences, Tabriz, Iran \\ ${ }^{4}$ Tabriz University of Medical Sciences, Tabriz, Iran \\ ${ }^{5}$ Cancer Registry Office, Liver and Gastrointestinal Diseases Research Center, Tabriz University of Medical Sciences, Tabriz, Iran \\ ${ }^{6}$ Tabriz Health Services Management Research Center, Tabriz University of Medical Sciences, Tabriz, Iran
}

Correspondence should be addressed to Saeed Dastgiri; saeed.dastgiri@gmail.com

Received 19 August 2020; Accepted 19 August 2020; Published 22 February 2021

Copyright (c) 2021 Roya Dolatkhah et al. This is an open access article distributed under the Creative Commons Attribution License, which permits unrestricted use, distribution, and reproduction in any medium, provided the original work is properly cited.

In the article titled "Breast Cancer Survival and Incidence: 10 Years Cancer Registry Data in the Northwest, Iran" [1], there was an error in "Figure 1(a)." The $p$ value has been corrected from $p \log -$ rank $=0.0169$ to $p \log -\operatorname{rank}=0.0692$. The corrected figure is shown below. 


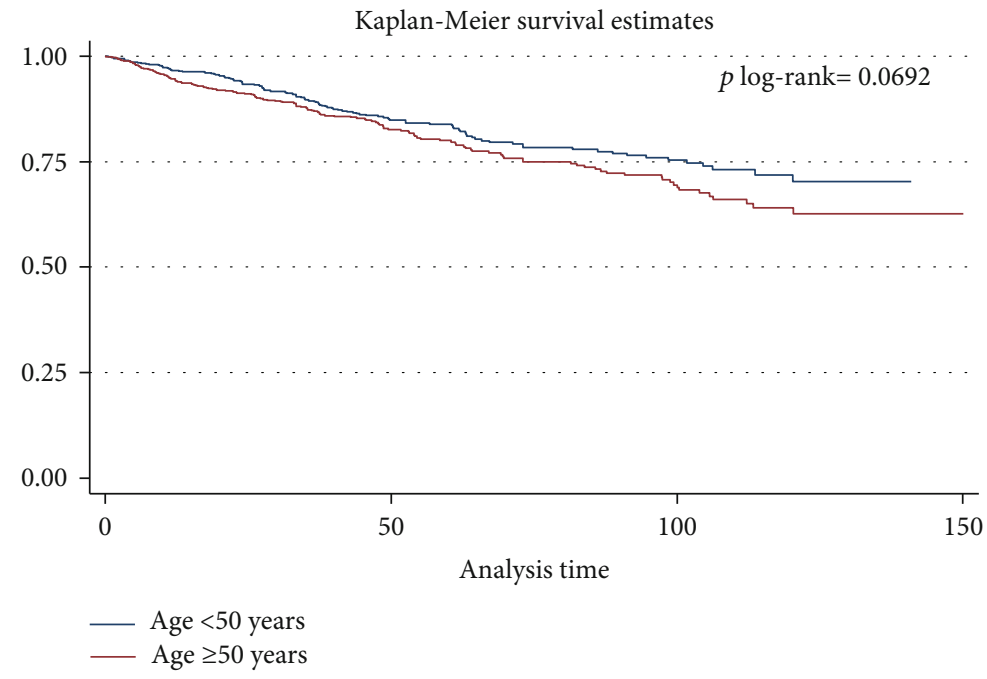

Figure 1: Kaplan-Meier survival curve, results for the test of equality of breast cancer-specific survival functions for the different variables in Northwest of Iran, between 2007 and 2016 in both sexes (a) age groups.

\section{References}

[1] R. Dolatkhah, M. H. Somi, M. A. Jafarabadi et al., "Breast Cancer Survival and Incidence: 10 Years Cancer Registry Data in the Northwest, Iran," International Journal of Breast Cancer, vol. 2020, Article ID 1963814, 6 pages, 2020. 